\title{
Pour une Critique des Médias
}

Écho

For a Media Critique. Echo

Jean-François Tétu

\section{CpenEdition}

\section{Journals}

Édition électronique

URL : http://journals.openedition.org/questionsdecommunication/7712

DOI : 10.4000/questionsdecommunication. 7712

ISSN : 2259-8901

\section{Éditeur}

Presses universitaires de Lorraine

Édition imprimée

Date de publication : 1 décembre 2006

Pagination : 247-262

ISBN : 978-2-86480-828-2

ISSN : $1633-5961$

Référence électronique

Jean-François Tétu, «Pour une Critique des Médias », Questions de communication [En ligne], 10 | 2006 mis en ligne le 01 décembre 2006, consulté le 22 mars 2021. URL : http://journals.openedition.org/ questionsdecommunication/7712 ; DOI : https://doi.org/10.4000/questionsdecommunication.7712 


\section{$>$ ÉCHANGES}

JEAN-FRANÇOIS TÉTU

Laboratoire Médias et identités

Institut d'études politiques de Lyon

Jean-Francois.Tetu@univ-lyon2.fr

\section{POUR UNE CRITIQUE DES MÉDIAS. ÉCHO}

Résumé. - Écrite en écho au texte de Daniel Dayan (2005), cette contribution repose sur l'analyse de la couverture, par la presse écrite française, de la seconde Intifada (2000-2002) et de la guerre au Liban (juillet-août 2006). Elle tente de mettre en lumière les répétitions et stéréotypes qui, dans la presse, opposent les civils (palestiniens puis libanais) aux soldats (israéliens) et absolvent les victimes de toute responsabilité attribuée, d'abord, au seul État israélien, avant que le terrorisme ne vienne brouiller les cartes. Mais l'image du militaire, omniprésente, tend à occulter celle du terroriste, si bien que la compassion, instaurée comme figure centrale du récit, empêche la compréhension du conflit. S'appuyant sur des effets de réel, elle ne cesse de dramatiser un conflit interminable qu'elle semble avoir renoncé à expliquer.

Mots clés. - Seconde Intifada, guerre israélo-libanaise, presse écrite, stéréotypes, compassion, victimes, information, mythe. 
Scepticisme, stupéfiance, incrédulité. Tels sont, à l'égard des médias, et plus particulièrement de la télévision, les sentiments dominants des citoyens. Chacun, confusément, sent bien que quelque chose ne va pas dans le fonctionnement général du système informationnel. Les mensonges et les mystifications de la guerre du Golfe - "I'lrak, quatrième armée du monde", "la marée noire du siècle", "une ligne défensive inexpugnable", "la guerre chirurgicale", "l'efficacité des Patriot", "le bunker de Bagdad", etc. - ont choqué, une fois encore, les téléspectateurs qui ont senti leur subjectivité se fracturer; cela a confirmé ad nauseam l'impression forte de malaise qu'avaient déjà suscitée des affaires comme le faux charnier de Timisoara, et la profanation de Carpentras $\gg$ '. Vieux de 15 ans déjà, cet éditorial d'Ignacio Ramonet, dans Le Monde diplomatique, montrait l'étendue d'un « soupçon » sur le journalisme qui n'a pas vraiment cessé depuis, même si la « déontologie » ou l'« éthique » journalistique est devenue, pendant cette période, un leitmotiv obligé. L'interview de Daniel Dayan (2005) par Béatrice Fleury et Jacques Walter, «Pour une critique des médias 》, vient à point nommé pour reprendre des questions « classiques » (objectivité, normes professionnelles, rapport de l'information à la vérité) qu'une bonne quinzaine d'années de ce « soupçon » ont maintenues comme très actuelles. II est difficile de savoir si la perte de crédit accordé au journalisme constitue la première cause de la désaffection croissante des lecteurs, un peu partout dans le monde, puisque la baisse régulière et continue du lectorat des quotidiens depuis la dernière décennie a peu affecté la diffusion des news, qui souffrent pourtant des mêmes maux, ni empêché la naissance d'une presse gratuite de qualité très variable, qui n'en est pas davantage exempte. Et les multiples critiques de la couverture télévisuelle, de la guerre du Golfe puis de la guerre contre l'Irak, n'ont pas fait diminuer de façon significative l'écoute des journaux télévisés, qui demeure la première forme d'information pour la population. En revanche, on voit bien que l'actualité fourmille d'excès en tous genres (la place accordée au « coup de tête » de Zinedine Zidane à la fin du Mondial 2006 de football), de retournements de perspective ahurissants (voir le feuilleton Arcelor-Mittal Steel et le « patriotisme économique »), ou de défaut radical d'explication (le rôle du pétrole au Darfour étant évacué, par exemple, au profit de considérations ethniques douteuses, quarante ans après le même détournement de l'information sur le Biafra). Il est donc nécessaire de reprendre ces « vieilles » questions, et on ne peut que remercier Daniel Dayan de nous inciter à réfléchir avec lui sur les liens actuels entre le récit et le rite, le rôle de l'information sur ce conflit, les cadres de l'interprétation journalistique, le fonctionnement de l'image télévisée. Ne pouvant traiter de tout cela, nous suivrons néanmoins quelques-unes de ces pistes dont la dernière guerre au Liban indique la brûlante actualité.

' I. Ramonet, « L'ère du soupçon », Le Monde diplomatique, avr. 199I. 


\section{La « couverture » du conflit israélo-palestinien, de la seconde Intifada à la guerre du Liban}

La question que pose Daniel Dayan est de savoir comment certains discours médiatiques sont possibles. Nous avons appris de Michel Foucault que les formations discursives sont le fait d'une foule d'interactions qu'on peut résumer en disant que l'information n'est pas circonscrite à l'action des seuls journalistes. Ainsi a-t-on pu expliquer que les dérives de l'information sur la Guerre du Golfe étaient l'effet direct des conditions de sa production. Daniel Dayan prend comme point de départ la couverture de la seconde Intifada, dont nous avions fait il y a quelques années une analyse un peu différente sur la seule presse écrite (Garcin-Marrou,Tétu, 2003). De cette étude, à laquelle nous ne pouvons que renvoyer, nous souhaitons cependant reprendre rapidement quelques points, mais il nous faut d'abord relever que les arguments usuels pour expliquer les dérives de l'information sur des conflits lointains sont ici inopérants : en effet, il y a un nombre important de journalistes sur place, leur circulation est à peu près libre, l'accès aux sources ne pose pas de problème, et les directions ne mesurent pas chichement l'espace ou le temps consacrés à ce sujet.

Le premier trait constitutif de ce conflit est son extrême complexité. II constitue le principal point commun de sa couverture par la presse, malgré la construction de catégories interprétatives à peu près constantes, toujours rapides, faites d'images et de rôles qui semblent se mettre automatiquement en place à chaque nouvel épisode : elles se répètent comme si la reconnaissance des motifs identiques assurait une meilleure compréhension des nouveaux épisodes de ce qui est présenté comme le «même » conflit. Ainsi, pour ne prendre qu'un exemple illustrant cette propension à la répétition des « motifs », la « riposte » d'Israël aux « provocations » du Hamas ou du Hezbollah apparait-elle toujours immédiatement comme « excessive » ou « disproportionnée ». C'est le thème commun de tous les commentaires de début juillet 2006 sur les premiers bombardements de l'armée israélienne au Liban. Mais nous n'avons trouvé aucun commentaire ou analyse du nom donné par Israël aux attaques qui commencent le 12 juillet 2006, «Punition adéquate », en réponse à l'enlèvement des deux soldats israéliens par le Hezbollah, qualifié par lui de "Promesse tenue ». II y aurait tout de même de quoi s'interroger sur cet « adéquat », et sur cette « promesse ». En effet, depuis des mois, le Hezbollah envoyait des roquettes sur la population civile du nord d'Israël (à l'intérieur des frontières de 1948), ce qui, en droit international, s'appelle « crime de guerre », et la dernière offensive du Hezbollah se situait sur ce territoire. Donc, alors même qu'il n'y avait eu aucune attaque du sol israélien (dans ses limites reconnues par la communauté internationale) depuis 1948, 
hormis quelques rares missiles irakiens pendant la Guerre du Golfe, la presse française a unanimement dénoncé cet « excès », comme s'il était un trait distinctif et constant de l'attitude israélienne. Comment comprendre cela?

D'abord, il est impossible de ne pas souligner la « neutralité impossible » des médias français dans ce conflit, assez remarquablement analysée lors d'une conférence tenue à Radio France le 25 avril 2002, sur le thème «Pour mieux comprendre le conflit israélo-palestinien »². En effet, les médias français ont été régulièrement accusés d'être pro-palestiniens, ce qui impose une réflexion préalable sur le tournant de 1967 et l'occupation des «territoires ». Jusque-là, l'opinion française et les médias avaient toujours été fortement pro-israéliens. Mais, en 1967, tout change, et Israël, menacé depuis ses origines, devient soudain l'« occupant » de territoires qui ne lui appartiennent pas. Ainsi le propos du général de Gaulle sur ce « peuple sûr de lui et dominateur » a-t-il renforcé le renversement de l'image de la victime devenue bourreau. Mais cela ne suffit pas à expliquer cette sorte de passion, ni la constance ou l'ampleur de la couverture médiatique : contrairement à la plupart des lieux de conflits où le nombre de journalistes est généralement très réduit (il n'y a plus un seul journaliste français en Côte d'Ivoire au nord de Bouaké depuis 2004, et il ne doit pas y en avoir beaucoup en Irak depuis la libération de Florence Aubenas), il y a ordinairement environ 300 journalistes étrangers présents à Jérusalem depuis le début de la seconde Intifada, ce qui est énorme ; il y en avait 900 au printemps 2002, aux pires moments de l'Intifada ! Pourquoi? Trois raisons sont généralement invoquées : le caractère démocratique d'Israël et la libre circulation des informations ; le fait que ce sol est un berceau de notre civilisation ; l'importance, en France, de la communauté juive et des communautés arabo-musulmanes. D'où la difficulté de la couverture du conflit, à la fois impérative et très difficile, sur laquelle insistent la plupart des journalistes concernés. Mais il y a au moins, dans ce conflit, un a priori permanent, une vulgate faite d'interprétations préalables. Une des plus importantes, comme on le verra, est une sorte de prime au faible contre le fort : " cette image se retournera toujours contre le char qui tire sur des gens armés de kalachnikovs $»^{3}$. La complexité de la situation incite donc les médias à tenter une réduction du conflit à des questions apparemment simples: celle de la responsabilité/culpabilité des protagonistes, elle-même fortement réduite à celle des victimes, et, de ce fait, à celle de la légitimité ou de l'illégitimité des attentats et des réactions violentes de l'armée, focalisant l'attention sur les épisodes les plus visiblement violents du conflit. Dans l'étude que nous avons conduite sur

\footnotetext{
${ }^{2}$ Cette conférence a été publiée dans Médias, 2, juin-juil. 2002, pp. 60-69.

${ }^{3}$ B.Vannier, directeur de la rédaction de France Inter, in : Médias, 2, juin-juil. 2002, p. 63.
} 
la seconde Intifada, nous avons tenté de repérer différents modes discursifs dans la représentation de la crise. Le premier, très visible dans les jours qui suivent la mort du petit Mohamed Al-Durah (30 septembre 200 I), est que les civils palestiniens sont représentés comme victimes constantes du conflit : ce sont toujours de « jeunes Palestiniens » qui sont face à des « militaires israéliens 》, qui « jettent des pierres » et reçoivent en réponse des « tirs à balles réelles ». La terminologie employée insiste systématiquement sur la disproportion entre les «manifestants » et les « soldats », dans le comportement comme dans le nombre des tués et des blessés ; en deux mots, le cadrage discursif oppose les civils aux soldats, les enfants aux adultes, en une dichotomie régulière qui met en cause la légitimité de la violence israélienne. Et s'il arrive que ces civils se livrent à une violence déchaînée, comme le lynchage d'un soldat réserviste dans un commissariat ( 12 octobre 200 I), c'est par une sorte de fatalité contre laquelle - d'ailleurs - l'Autorité palestinienne est impuissante, ce qui altère sa crédibilité. Une évolution sensible apparaissait cependant : aux débuts de la seconde Intifada, le terme de « terrorisme » était très peu utilisé ; lorsqu'il commence à l'être, il renvoie à une autre lecture du conflit, où les actes terroristes sont liés à un pouvoir palestinien ambivalent qui instrumentaliserait les souffrances de son peuple. Si bien que le terrorisme a déplacé la question de la légitimité : certes, la violence de la répression israélienne est disproportionnée, et en tant que telle, illégitime. Mais, l'État israélien est légitime et ne peut être qualifié de terroriste alors que, de l'autre côté, les actions violentes (des civils ou des milices) semblent illégitimes, et la police palestinienne, légitime, reste impuissante, si bien que c'est l'action du pouvoir palestinien qui commence à paraître illégitime. Ainsi peut-on affirmer sans hésitation que la lecture du conflit faite par la presse est singulièrement plus complexe que l'attitude de la télévision dénoncée par Daniel Dayan.

Ce déplacement de la lecture du terrorisme nous semblait lié aux attentats du II-Septembre qui ont, pour les journaux en tout cas, fortement modifié la perception du recours au terrorisme comme forme de « résistance », ce qui apparaissait assez clairement dans la couverture de l'anniversaire du début de l'Intifada, où l'on trouvait un double cadrage. D'un côté, un cadrage « pro-palestinien » qui rappelle les origines ponctuelles de I'Intifada (provocation d'Ariel Sharon), et les réactions disproportionnées de l'armée à l'égard d'une révolte née des frustrations du peuple palestinien : ce cadrage, qui insiste sur l'escalade guerrière d'Israël, repose toujours sur l'opposition entre le «peuple palestinien » et l'« État d'lsraël ». Mais c'est le refus du cadrage « peuple réprimé vs État répressif » qui est mis en cause par l'autre lecture, celle que proposait Le Monde par exemple, pour qui l'explosion populaire initiale a été très vite « militarisée » et « privatisée ». C'est 
pourquoi la figure centrale n'est plus, dès lors, et jusqu'en juillet 2006, celle de la victime, mais celle de l'échec de ce qui peut se définir comme stratégie (de Yasser Arafat ou d'Ariel Sharon), tactique (des milices, et spécialement du Hamas et du Hezbollah), ou plus généralement, action des Palestiniens. Et cela à cause de la « dérive » que représente le terrorisme. Évidemment, ce cadrage conduisait à faire du II-Septembre et de la lutte contre le terrorisme le cœur du commentaire : les Palestiniens doivent désormais choisir le bon camp en abandonnant le terrorisme. En outre, ce cadrage permettait une re-lecture des origines de l'Intifada et une mise en cause de l'Autorité palestinienne : retour des « accusations récurrentes de corruption et d'inefficacité », jusqu'à la mort de Yasser Arafat, et d'« impuissance » après la victoire du Hamas aux élections.

Le I I-Septembre a marqué une rupture majeure, repérable dans le changement des oppositions lexicales. Le cadrage lexical antérieur, on l'a dit, reposait sur l'opposition « peuple palestinien vs État israélien »; les actions militaires opposaient «les combattants palestiniens » à «l'armée israélienne » (dans ces deux cas, l'opposition portait de fait sur celle entre les « civil » et les « soldats »). De ce fait, avant le IISeptembre, l'emploi du vocable « terroriste » n'était pas totalement délégitimant. Le terrorisme était compréhensible parce que les médias français n'associaient pas l'acte terroriste, présenté comme individuel et désespéré, à une stratégie de l'Autorité palestinienne. Or, le IISeptembre a interdit la poursuite de ces oppositions, parce que le « terroriste » n'a plus de légitimité dans l'opinion, ce qui n'a fait que s'accroître dans les années suivantes. En outre, il a produit une collusion entre le caractère religieux et le caractère ethnique du conflit. Si, auparavant, le Hamas est seul à être continûment caractérisé comme " islamique », il n'y avait pas d'assimilation entre le mouvement palestinien, identifié à coup sûr comme « arabe », et un quelconque ensemble « islamique ». Désormais, la revendication « nationale » d'un État palestinien est susceptible d'être parasitée par le fantasme d'une guerre de religion, renforcée, en juillet 2006, par les enlèvements simultanés (et supposés coordonnés) de soldats israéliens par le Hamas, d'abord, puis par le Hezbollah, dont le nom indique bien la volonté ouvertement religieuse.

Ainsi la couverture par la presse des deux premières années de la seconde Intifada nous poussait-elle à un jugement beaucoup plus nuancé que celui de Daniel Dayan sur la télévision. À l'exception notable de L'Humanité qui s'en tenait à la condamnation de l'agression israélienne et à la colonisation des territoires, l'immense majorité des médias montrait une réprobation claire et de plus en plus nette des attentats, à la fois parce qu'ils font des victimes innocentes, parce qu'ils entretiennent une angoisse génératrice de représailles, et parce qu'ils ruinent les efforts de 
la diplomatie. Mais les journaux ne parvenaient toujours pas à rendre compte de la complexité de la situation (sans laquelle la violence des combats de Jénine est incompréhensible), préférant condamner nettement l'interdit touchant alors aux journalistes de circuler normalement. L'absence de « liberté » de l'information était dénoncée parce qu'elle autorisait toutes les rumeurs, et laissait supposer l'existence d'atrocités que démentait Israël. Au fond, disions-nous, la principale difficulté que les journaux rencontrent dans ce conflit est une sorte d'impasse narrative. Sauf à maintenir une grille interprétative très simplifiante (agresseurs vs victimes), il est impossible de construire des rôles actanciels clairs, et les figures semblent sans cesse brouillées : ainsi de l'attitude de la population, mais aussi des auteurs des attentats (fanatisme religieux ou résistance ?) et, bien entendu, des responsables politiques (sincérité de Yasser Arafat ? objectifs réels d'Ariel Sharon ?). Et ainsi de suite. La présence supposée de «terroristes 》 au milieu des civils brouillait l'image de l'asile franciscain de Bethléem, les contradictions des témoignages à propos du « massacre » de Jénine. De part et d'autre, il était alors plus facile de dénoncer l'effort de propagande, parce que la poursuite des attentats terroristes après le II-Septembre avait pour une part disqualifié dans l'opinion la résistance palestinienne, comme elle a fini de sceller l'alliance entre Israël et les États-Unis.

Au moment où nous écrivons ces pages, la guerre fait rage au Liban. Aux premiers jours de l'offensive israélienne, en riposte à l'enlèvement des soldats israëliens par le Hezbollah, la presse souligne donc surtout l'excès de la riposte, jugée globalement disproportionnée, mais pas la riposte elle-même, considérée comme justifiée. Certes, l'accent est mis, et de plus en plus, sur les destructions et les morts au Liban, mais les reportages sur les villes d'Israël touchées par les roquettes ne sont pas absents, et surtout, l'accent se déplace vers la nécessité d'un cessez-lefeu, vers le "drame humanitaire », et l'imprévisible issue du conflit. On voit donc le cadrage s'élargir, assez vite, vers la communauté internationale, et en particulier les États-Unis, tant il apparait que la volonté américaine de retarder le cessez-le-feu semble faire de l'offensive israélienne l'instrument des États-Unis. II reste que la « disproportion 》 est sans cesse renforcée par les données chiffrées : en juillet 2006, 27 civils israéliens tués, et 900 « personnes 》 au Liban, 900000 personnes déplacées (sur environ 4 millions) ; pour la seule journée du 3 août, il est tombé environ 200 roquettes sur Israël, et 4000 obus au Liban, etc.

Ce nouveau contexte permet de reprendre la question initiale (comment parler de ce conflit ?) en comparant le traitement des deux périodes (200 I/2002 et juillet-août 2006). Premier constat : la difficulté des explications, commune à tous les conflits très durables, c'est-à-dire l'absence de tentative d'explication globale de la situation. À cet égard, on remarquera que les « grands reportages », dossiers, enquêtes, qui 
faisaient il y a quelques décennies encore la richesse des quotidiens, ont quasiment disparu. De même, la fonction de l'éditorial, qui permet de prendre une grande distance, a changé : ce n'est plus sous le point de vue de Sirius que l'éditorialiste parle, mais à une dist ance très proche. Si bien que le débat tend à disparaitre des articles des journalistes, pour se réfugier dans le courrier des lecteurs et toutes ces rubriques spécialisées, assez récentes, qu'on nomme « Débat », « Forum », « Rebonds », etc. où les opinions les plus opposées s'exposent, avec une volonté éditoriale d'équilibre, sur laquelle il convient de s'interroger : cette apparence d'objectivité, ou d'équilibre, renvoie-t-elle à une recherche de débat ou de vérité ?

À défaut de tenter une explication globale, probablement impossible, ou tenue pour impossible, on développe les causes immédiates (Pourquoi? Exemple : « Pourquoi nous ? », se demandent les chrétiens libanais) et les intentions supposées (Pour quoi ? Exemple : «Veut-on détruire le Liban ? »). Or, l'écrit bénéficie d'un avantage considérable sur l'image ou le son, car il peut juxtaposer, et par là même, argumenter.Voyons cela : le 12 juillet, soit juste avant l'offensive au Liban, il n'est encore question que du caporal Shalit, enlevé par le Hamas. Le Monde lui consacre une demi-page, en deux parties : à gauche en caractère romains gras, un énoncé du journal «Israël refuse l'échange de prisonniers proposé par le chef en exil du Hamas », et sous ce titre, sur trois colonnes, curieusement, la photographie d'une femme voilée tenant un drapeau blanc, et marchant sur une route déserte avec la légende « Après avoir fui devant l'armée israélienne, une Palestinienne tente de gagner un endroit sûr, dans le sud de Gaza ». Contraste assez fort, donc entre le « dialogue de sourds » des protagonistes dont parle l'article, et la déréliction d'une femme du peuple palestinien. À droite de la même page, un autre article, titré en italiques, indique typographiquement la rupture énonciative et le témoignage qui suit : " Mère d'un soldat israélien enlevé en 1994, Esther Waxman se souvient 》. On y apprend que le fils de cette femme, enlevé par le Hamas, a été tué lors de l'assaut lancé contre le repaire de ses ravisseurs. Que dit-elle ? « En tant que mère, j'aurais tout tenté pour revoir mon fils $[\ldots]$; en tant que citoyenne, je savais aussi qu'un pays doit respecter certains principes [...]. En 1985, I I 50 Palestiniens ont été relâchés en échange de trois de nos soldats. Dans la première Intifada, puis la seconde, des Israéliens ont payé de leur vie cette libération ». Et le journaliste d'ajouter, comme si le lecteur n'avait pas compris, que « son discours est ambivalent » avant de conclure sur le « deuil d'un fils aimé, tué un jour d'octobre 1994, dans une cache située à 10 minutes en voiture de son domicile ».

Que dire de cela? Par la photographie de la Palestinienne et le témoignage de l'Israélienne, le journal semble « équilibrer » les points de vue, mais, en fait, il fait bien autre chose : il prend le parti de la victime, 
ou plutôt de deux victimes, l'une palestinienne et l'autre israélienne, toutes deux victimes d'une sorte de fatalité. Et cela donne une première clé de la stratégie, ou de la logique du journalisme contemporain, singulièrement plus facile que de tenter de discuter des responsabilités, des causes et des fins. Apparemment, tout y est - la disproportion entre les prisonniers de chaque camp, l'existence du terrorisme palestinien, la proximité des adversaires (《 10 minutes 》), et la « fuite » de la Palestinienne (civile) devant l'armée (israélienne) -, mais ne conduit qu'à la compassion, et d'aucune façon, à la compréhension du conflit et de ses atrocités.

Pendant les trois premières semaines de l'offensive israélienne, la place des opérations militaires proprement dites est relativement réduite : chaque jour procure de nouvelles infographies avec leurs pictogrammes sur les lieux de bombardement ou d'impacts de roquettes, sur la nature des armements, sur le nombre d'immeubles détruits (et le coût de la reconstruction)... Mais de très loin, l'essentiel est - d'une part et d'abord - la situation des populations civiles (surtout libanaises, naturellement, vu l'ampleur des bombardements), et de l'autre, les jeux diplomatiques. Pour mieux comprendre la logique de ce discours, il faut regarder les photographies qui, curieusement, font se retrouver les mêmes oppositions que quatre ans plus tôt. Qu'y voit-on ? D'un côté, des soldats israéliens (avec leurs armes et leurs chars), et de l'autre, des civils libanais (à côté des ruines de leur habitation et des blessés ou des morts de leur famille). Et cette opposit ion penche toujours en faveur des civils dont le seul rôle est d'être victime. Cette opposition est-elle spécifique de la situation israélienne ? Oui, et non. Oui, parce qu'elle est omniprésente dans la représentation d'Israël depuis 1967. Non parce que c'est la même opposition qu'on retrouve en Irak depuis la fin officielle de l'offensive américaine. Et cela pousse à deux réflexions.

D'abord, les guerres modernes font beaucoup plus de victimes civiles que militaires, on le sait, si bien que l'image du militaire est plus ou moins confusément associée à l'agression. L'imaginaire social ne trouve qu'une seule guerre « juste » dans notre histoire, la guerre contre le nazisme. Ce pourquoi il fallait, en 1989, donner au communisme le visage du nazisme au moment de la chute de Ceauflescu, ou, deux ans plus tard, donner à Saddam Hussein les traits d'Hitler. Si bien que l'image du soldat est liée soit à l'agression (les Russes en Afghanistan ou en Tchétchénie), soit à la répression excessive (les Américains en Irak, et les Israéliens au Liban ou à Gaza). Ensuite, en face de ces soldats, depuis qu'il est terroriste, « l'ennemi » n'a pas de visage : on ne voit que des « civils » mais pas les tireurs de roquettes ou de missiles. Une exception, cependant, les 18 et 21 juillet 2006, dans Libération, où deux photographies de Bruno Stevens (Cosmos), prises le même jour « à Kfarchima, près de Beyrouth » montrent deux civils armés de fusils 
d'assaut, l'un barbu, l'autre imberbe, avec une légende presque identique : «Un membre du Hezbollah » (avec le lieu et la date imprécise que nous pensons être le 17). Nous relevons cette légende qui ne qualifie pas ces hommes puissamment armés de « soldat » ou de « combattant », ce qu'ils sont pourtant.

Cela explique aussi l'ampleur de l'onde de choc du I I-Septembre : il n'y avait que des victimes, civiles, innocentes, et pas d'ennemi immédiatement identifiable, si bien que l'attentat, faisant surgir une menace potentiellement omniprésente, mais invisible, soudait la population dans le sentiment de la crainte et de l'horreur. La question que pose Daniel Dayan peut alors prendre la forme suivante: «Comment le directeur d'un grand journal français peut-il écrire : nous sommes tous américains ? » La réponse est, pensons-nous, directement liée à l'image contemporaine de la victime qui, parce que victime, ne saurait être coupable. C'est cela qui nous semble pouvoir expliquer le très curieux comportement global des médias dans le conflit israélopalestinien : les attentats perpétrés par les Palestiniens ne provoquaient pas le rejet qu'ils auraient dû produire. Pourquoi ? Peut-être parce que les Palestiniens, déjà victimes (de la perte de leur sol, d'une répression violente, de conditions de vie difficiles, etc.) ne sauraient être « vraiment » coupables, alors qu'lsraël, déjà coupable (de l'occupation des « territoires », d'une répression violente et toujours décrite comme 《 excessive » ou « disproportionnée », etc.) ne saurait être « vraiment 》 victime. Nous en voyons une illustration assez flagrante dans l'éditorial de Libération du 9 août : En menant une telle guerre ouverte au Liban, avec son cortège de victimes civiles, Israël a réussi le tour de force de passer aux yeux du monde de victime à agresseur, à faire du Hezbollah le héros ambigu du monde arabe, Palestiniens compris 》.

Cela semble constituer comme un écho très lointain de l'histoire même de ce conflit : la déclaration Balfour ( 12 novembre 1917) ne parlait que des droits civiques et religieux des non juifs, et pas de leurs droits politiques : et les Arabes de Palestine, qui représentaient alors $90 \%$ de la population, n'étaient pas même nommés. Et les mêmes Arabes de Palestine n'eurent pas leur mot à dire sur le partage de la Palestine de 1948, ni sur l'armistice de 1949 entre Israël et les quatre États arabes voisins. Cela n'est quasiment jamais rappelé, mais fait partie d'une sorte de mémoire collective qui assure le « cadrage » du conflit. II en va de même avec le Liban « grand ami de la France »: pourquoi « grand ami »? Est-ce pour cela que les médias français ne reprochent pas vraiment au Liban de n'avoir pas appliqué la résolution I 559 du Conseil de sécurité de l'ONU alors qu'ils rappellent plus souvent qu'Israël n'a pas appliqué les résolutions qui lui enjoignent de quitter les territoires ? 
Il y a bien deux poids et deux mesures. Ils doivent peu à la connaissance de l'histoire du conflit, rarement rappelée. Certes, au moment de la victoire du Hamas aux élections, I es médias ont insisté sur le danger de ce scrutin, du fait de la volonté avouée du Hamas de détruire l'État d'Israël, mais ils ont fait peu de liens, avant juillet 2006, sur la double menace simultanée du Hamas et du Hezbollah que les enlèvements semblaient faire presque découvrir. Et enfin, dans ce cas précis, ils s'interdisent de voir contrairement à la lecture qu'ils font des États-Unis, que ce terrorisme, conduisant à la restriction des libertés publiques, atteint son but, miner le fonctionnement de la démocratie.

\section{Le récit et le rite}

Dans l' émission Arrêt sur image (16/05/04), Daniel Dayan a suggéré que la ritualisation de la mise en scène de l'exécution d'un otage américain en Irak fonctionnait comme un retournement horrifiant du sacrifice d'Abraham. Si, pour ses auteurs, la mise en scène de cet assassinat indique bien que cette mise en scène est rituelle, sacrificielle, il est nécessaire de $s$ ote interroger aussi sur ce qui, de l'information, bascule vers le rite ou le mythe. Reprenant le terme d'« infamie », utilisé par un journaliste du Monde, Daniel Dayan dénonce l'expression de « critiques sur le mode de la ferveur ». On ne peut que le suivre sur ce point, car on s'aperçoit que la rationalité spécifique mise en œuvre dans le discours médiatique est fon damentalement dirigée vers l'émotion. Nous avons assez répété que la logique discursive était dominée par la compassion à l'égard des victimes, et que cette compassion était la matrice du discours sur ce conflit. Pour comprendre cette façon de parler, il faut donc opposer le « savoir de connaissance » au « savoir de croyance » qui est à l'œuvre lorsqu'on vise l'émotion. À l'origine, il y a bien une information, c'est-à-dire un savoir. Mais, loin que ce savoir repose sur des critères de vérité extérieurs au sujet (savoir de connaissance), il est tout aussitôt évalué, condition indispensable pour l'émergence de l'émotion ; c'est-à-dire qu'il se structure autour de valeurs socialement partagées (il faut pouvoir les « reconnaitre »), mais subjectivement investies ; plus exactement, ce savoir est polarisé autour de valeurs (pitié ou indignation, par exemple), socialement acceptées du fait de la construction des rôles auxquelles elles sont associées. La construction narrative - distribuant les rôles et les figures - est le premier moteur de cette « croyance ». Mais le récit n'a pas d'autre fin que fantasmée : la paix impossible.

La particularité de la situation médiatique est que là où, dans la réalité, il y a urait par exemple une obligation morale d'assistance, il ne peut y avoir pour l'usager des médias qu'une saturation de l'imagination. Et, bien entendu, l'image télévisée en joue infiniment plus que la distance de 
l'écrit, notamment en ce qui concerne toutes les images « en direct», qui semblent faire surgir le réel (au sens lacanien), au lieu de construire une représentation et une signification. II ne reste à l'usager des médias que l'image de lui-même, confrontée à ce qu'il ne peut assimiler que comme fantasme. Or, dans le récit actuel de l'offensive israélienne, les journaux insistent beaucoup sur le «patriotisme » ou le « nationalisme » libanais, lequel s'exprime fréquemment sous la forme d'un attachement renforcé (pour les chiites), ou totalement nouveau (chez les autres) au Hezbollah. Et les journaux insistent sur le dénuement des gens qui ont fui leurs villes et leurs villages, ce qui est parfaitement compréhensible. Mais on y voit aussi fréquemment la mention du fait que les Libanais, ayant trouvé un abri, suivent sur les écrans de télévision les récents développements de l'offensive israélienne. Or, il est rare que les reporters disent ce qu'ils y voient. Examinons ceci. Si la chaîne qatari AlJazira, et celle fortement soutenue par les américains - Al Arabiya multiplient, en un réflexe « professionnel », les divers points de vue, la chaîne la plus regar dée est bien la chaîne chiite Al Manar, qui continue d'émettre, malgré le bombardement de l'immeuble qui l'abritait. Or cette dernière, toute dévouée à Hassan Nasrallah et au Hezbollah, ne cesse de montrer des images terrifiantes - cadavres d'enfants, hurlements de douleur, etc. - mêlées sans vergogne aux images d'archives de Sabra et Chatila, et aux exhortations de Nasrallah ou de quelque imam (en général plutôt l'émir Fadlallah) : « À l'instant même où les Israéliens franchissent nos frontières, l'heure du djihad a sonné. Lavez alors l'honneur de l'Islam, lavez l'honneur des musulmans ». Prompts à dénoncer la souffrance indiscutable du peuple libanais (voir l'usage par Israël des sous-munitions), nos journaux s'interrogent assez peu sur les manipulations religieuses et racistes de l'opinion locale.

Ce basculement d'un conflit, dont les origines sont territoriales, vers l'expression constante d'une haine antisémite (côté libanais), couplée à un conflit religieux, produit une inflexion sensible dans le discours à partir de début août, si bien que les rôles semblent se brouiller encore davantage. En tém oigne l'éditorial de Libération, le 4 août, qui parle du chef du Hezbollah comme du "nouveau héros d'une "rue arabe" prompte à suivre des chimères [mis en italiques par nous] »: ces derniers mots tranchent singulièrement avec le ton usuel de ce journal lorsqu'il évoque les populations civiles arabes. Si ce type de propos indique une inflexion sensible pendant l'été 2006, il reste que, globalement, c'est ce savoir de croyance qui l'emporte. II repose d'abord sur un récit, puis sur un lexique où les « formules » font florès. On l'a $\mathrm{vu}$, le récit repose sur l'articulation des actants autour d'un objet de valeur, socialement partagé, et l'investissement thématique dans la construction de figures (les personnages) qui lui donnent l'épaisseur du réel. Or, la répétition inlassable des mêmes personnages dans les mêmes 
rôles (peu ou pas de transformations dans les parcours figuratifs) produit deux choses distinctes : le sentiment d'une impasse totale dans le conflit, et celui d'une sorte de fatalité qui s'abat sur les personnages condamnés à revivre les mêmes rôles. Le tout dans un macro-récit où la signification propre de chaque épisode (telle réponse à telle provocation, à tel moment), disparait derrière la fixité des rôles dont l'archétype est la brutalité ou les excès d'Israël contre la déréliction du peuple palestinien. Nous ne disons pas que cela n'est pas, mais que cela constitue le mécanisme même du mythe, tel que le décrivait Roland Barthes (1957).

Dans la réalité, la situation émouvante tient à la nature de la relation entre le sujet et cette situation, qui provoque peur ou colère, joie ou honte, etc. Le support d'information doit évoquer quelque chose qui soit commun au groupe social. II lui faut représenter une situation à propos de laquelle une norme sociale (un jugement de valeur socialement partagé) met en cause, dans un récit, un actant qui est, le plus souvent de façon soudaine, victime ou bénéficiaire. En outre, cette situation doit reposer sur une motivation forte. Comme nous savons tous que l'émotion ne va pas sans les sentiments qui l'accompagnent ou la suivent, c'est par le brandissement des signes exprimant les sentiments que la situation est rendue émouvante : « Le monde a peur » titrait Le Parisien, le 12 septembre 200I. Et comme nous avons appris, depuis l'enfance, que les sentiments peuvent se communiquer plus facilement lorsqu'on en amplifie les signes, la «dramatisation » est une constante de la médiatisation de l'émotion, sous des formes évidemment propres à chaque support. La dramatisation des sentiments est donc le premier ressort thématique de la représentation émotionnelle du conflit. Le second - qui va de pair avec lui au point d'en être quasiment indistinct est à coup sûr son événementialisation, c'est-à-dire une « fracture dans l'état du monde ». Des expressions comme « L'offensive d'Israël ravage le Liban » ou, après le sur-titre « Sud Liban », avec la recherche d'un vocabulaire minimal des titres de Libération, « Terre brûlée », indiquent la volonté des informateurs de mettre le public dans une sorte de prise directe sur l'événement, qui conduit à brandir en titre, tantôt une posture compassionnelle ( « la peur »), tantôt la dénonciation des bourreaux («Terre brûlée »). Ce sont là les deux grands régimes de discours qu'on retrouve chaque fois qu'une « catastrophe » alimente l'actualité : Tsunami, marée noire, etc. Chaque fois, on trouve trois composantes majeures : la norme sociale sous forme de morale à maintenir (solidari té à l'égard des victimes vs dénonciation des bourreaux ou de la fatalité), ici l'impératif humanitaire ; la norme sociale sous forme de l'« opinion », prompte à dénoncer l'inaction des grandes puissances, ici l'inefficacité des résolutions onusiennes, et l'impuissance de la FINUL ; l'esthétisation du spectacle de la désolation : pietà pale stinienne, ou libanaise, à la suite d'un massacre ou d'un bombardement (mais aussi des attentats suicides). 
La compassion et la dénonciation (Boltanski, 1993) peuvent prendre d'autres formes : celle de l'attendrissement devant l'action bienfaisante (l'évacuation des ressortissants français du Liban), et, à l'opposé, celle de l'indignation qui présuppose l'existence d'un monde fondamentalement juste (bombardement de Cana). En outre, l'appel à la justice permet d'articuler la dénonciation du malheur et l'accusation des auteurs présumés du même malheur : la folie des hommes, ou leur inhumanité, ce qui explique les figures majeures du thème de la pitié.

Une caractéristique nette de la mythification du récit est la « croisade des vocabulaires » dont parle Daniel Dayan. Ces leitmotiv lexicaux sont des « formules », au sens où Jean-Pierre Faye (1972) utilise cette notion à propos des discours italiens et allemands sur «l'État total », reprise par Pierre Fiala et Marianne Ebel (1983) sur la xénophobie en Suisse, et longuement analysée par Alice Kieg-Planque (2003) au sujet de la «purification ethnique » dans l'ex-Yougoslavie : la formule, selon JeanPierre Faye (1972 : 38), est un « sur-récit » (un récit des récits), qui « prend en compte les fragments de récit, et les désarticule, tout en les réarticulant sur un autre niveau $\gg$. La force de la formule est qu'elle devient un passage obligé du discours : dans une tout autre contexte, la «fracture sociale » ou la «mondialisation » ne fonctionnent pas autrement. Ainsi, dans ce récit, le «massacre » et le « carnage » (d'ailleurs susceptibles d'être utilisés après un bombardement comme après un attentat-suicide), et des qualifications figées comme «le boucher de Chatila et Sabra ». Chacun sait de quoi on parle, et on peut « reprendre » inlassablement la formule qui ne cesse de circuler. Évidemment, notre propos n'est pas d être, mais de dicto. II s'agit là d'« événements de discours »: «Une séquence est constituée en événement de discours lorsqu'elles est réflexivement notoire, temporellement marquée, et dotée d'une pertinence dans le cadre de son surgissement et, partant, provoque des réactions (en pensée, en parole, en action) » (Krieg-Planque, 2003 : 309). Généralement, la formule est reprise avec un contingent de déterminants (« horrible », « terrible », etc.) qui semblent accentuer la référence en masquant le figement de la formule. C'est la porte ouverte au discours mythique de deux façons : d'abord, parce que la référence semble disparaître (ou se résumer) dans la formule : tout est dit. Ensuite, parce que les « victimes », tout comme leurs « bourreaux », sont privés de leur histoire propre qui leur est ainsi « volée », pour parler comme Roland Barthes. Mais, dira-t-on, comment parler ou écrire autrement? En cessant de restituer des bribes de propos, de scènes, et, pour commencer, en tentant de resituer ces propos et ces scènes. En premier lieu, c'est l'image et ses légendes, ou ses commentaires, qui portent la principale responsabilité de cette «mythification »: ainsi les légendes du Monde indiquant, le 20 juillet : « Des artilleurs israéliens tirent sur des cibles situées en territoire libanais [...] », ou, le 4 août, « Des soldats 
israéliens de retour d'opérations au Liban sud, mardi |er août ». Ces soldats sont à jamais privés de leur histoire et de leur identité, comme ce « un rapatrié » du même 20 juillet (p. 3).

\section{Conclusion}

Daniel Dayan pointe deux pathologies de la lisibilité de l'image, mais il faudrait ici reprendre tout ce qui a été écrit par de multiples auteurs sur l'image et notamment l'image d'information. On ne le dira jamais assez : si l'écrit peut argumenter et se fonde sur des signes, l'image se contente de montrer ce qui constitue moins des signes que des insignes. L'image est fondamentalement continuiste, d'où son lien étroit avec l'inconscient, où la contradiction n'existe pas. On l'a dit et répété : l'image ne sait pas nier, ni interroger, elle n'enregistre ni les flexions temporelles, ni leurs modes : pas de duratif, ni de passé, ni de futur, ni d'optatif, ou à plus forte raison d'irréel dans l'image. L'image est toujours particularisante (l'exemplaire y est pris pour le genre), et, comme elle est toujours une trace (le fameux « avoir été là » de Roland Barthes), elle modifie le rapport au croire, puisqu'elle nous en donne comme la preuve. Mais surtout, l'image ignore superbement toutes les catégories logiques, les subordinations, les concessions, les hiérarchies logiques : comment dire avec une image : si (le Hezbollah prend des soldats en otage), alors (il y aura une riposte) ou : si (Israël multiplie les colonies sur un sol initialement destiné aux Palestiniens) alors, il y aura des actions violentes. Comment dire à l'image « ou bien... ou bien » ou encore : « ceci, sauf... », etc. L'impasse où est engluée la représentation de ce conflit n'est pas seulement une impasse narrative, mais celle des stéréotypes où l'opposition binaire hallucinante entre les axes du « Bien » et du « Mal » ne conduit qu'au renforcement des affects, au détriment des significations.

\section{Références}

Barthes R., 1957, Mythologies, Paris, Éd. Le Seuil.

Boltanski L., 1993, La souffrance à 8 distance. Morale humanitaire, médias et politique, Paris, Métailié.

Dayan D., 2005, « Pour une critique des médias », Questions de communication, 8, pp. 195-222.

Faye J.-P., 1972, Langages totalitaires, Paris, Hermann.

Fiala P., Ebel M., 1983, Langages xénophobes et consensus national en Suisse : discours institutionnels et langage quotidien; la médiatisation des conflits, Neufchâtel, Université de Neufchâtel.

Foucault M., 1969, L'archéologie du savoir, Paris, Gallimard. 
Garcin-Marrou I., Tétu J.-Fr., 2003, « Seconde Intifada et terrorisme. Une analyse des discours de la presse française », pp. 849-863, Annuaire Français de Relations Internationales, vol. IV, Bruxelles, Bruylant.

Krieg-Planque A., 2003, « Purification ethnique ». Une formule et son histoire, Paris, CNRS Éd. 